\title{
Modeling and Analysis of an Epidemic Model with Non-monotonic Incidence Rate under Treatment
}

\author{
T. K. Kar (Corresponding author) \\ Department of Mathematics \\ Bengal Engineering and Science University, Shibpur \\ Howrah-711103, West Bengal, India \\ E-mail:t_k_kar@yahoo.com, tkar1117@gmail.com \\ Ashim Batabyal \\ Department of Mathematics \\ Bally Nischinda Chittaranjan Vidyalaya, Bally Ghoshpara, Howrah \\ West Bengal, India \\ E-mail: ashim.bat@gmail.com
}

\begin{abstract}
An epidemic model with non-monotonic incidence rate under a limited resource for treatment is proposed to understand the effect of the capacity for treatment. We have assume that treatment rate is proportional to the number of infective when it is below the capacity and is a constant when the number of infective is larger than the capacity. Existence and stability of the disease free and endemic equilibrium are investigated for both the cases. Some numerical simulations are given to illustrate the analytical results.
\end{abstract}

Keywords: Epidemic, Endemic equilibrium, Treatment, Basic reproductive number, Limit cycle

\section{Introduction}

The incidence in an epidemiological model is the rate at which susceptible become infectious. The form of the incidence rate that is used in the classical Kermack Mckendrick model (1927) is the simple mass action $\lambda S I$ where S and I denote the number of susceptible and infectious, respectively, $\lambda$ is called the infection coefficient. The standard incidence is $\lambda S I \mid N$, where $\mathrm{N}$ is the total population size and $\lambda$ is called the daily contact rate. Another kind of incidence is the saturation incidence $\lambda S I /(c+S)$ where $\mathrm{c}$ is a constant. When the number of susceptible $S$ is large compared to c that incidence is approximately $\lambda I$. This kind of incidence was proposed by Anderson and May (1979), Lourdes and Matias (1991). Many researchers (see Hethcote and Levin, 1988; Esteva and Matias, 2001; Liu et al., 1986; Liu et al., 1987) have proposed transmission laws in which the nonlinearities are more than quadratic. Ruan and Wang (2003) studied an epidemic model with a specific nonlinear incident rate $\lambda I^{2} S /\left(1+\alpha I^{2}\right)$ and presented a detailed qualitative and bifurcation analysis of the model. They derived sufficient conditions to ensure that the system has none, one, or two limit cycles and showed that the system undergoes a Bogdanov-Takens bifurcation at the degenerate equilibrium which include a saddle-node bifurcation, a Hopf bifurcation, and homoclinic bifurcation.

A more general incidence rate $\lambda I^{p} S /\left(1+\alpha I^{q}\right)$ were proposed by many researchers and authors (see, Liu et al., 1986; Derrick and Van den Driessche,1993; Hethcote andVen den Driessche, 1991, Alexander and Moghadas, 2004). Xiao and Ruan, 2007 proposed an epidemic model with non-monotonic incidence rate $\lambda I S /\left(1+\alpha I^{2}\right)$. Treatment plays an important role to control or decrease the spread of diseases such as flue, tuberculosis, and measles (see Feng and Thieme, 1995; Wu and Feng, 2000; Hyman and Li, 1998). In classical epidemic models, the treatment rate is assumed to be proportional to the number of the infectious, which is almost impossible in real perspective because in that case the resources for treatment should be quite large. In fact, every country or society should have a suitable capacity for treatment. If it is too large, the country or society pays for unnecessary cost. If it is too small, the country or society has the risk of the outbreak 
of a disease. Wang (2006) proposed a treatment function:

$$
\begin{aligned}
T(I) & =r I, \text { if } 0 \leq I \leq I_{0}, \\
& =K_{1}, \text { if } I>I_{0},
\end{aligned}
$$

where $K_{1}=r I_{0}$. This type of treatment function is more realistic because in every hospital, the number of beds is limited and also they have a certain capacity of medicines. In our proposed model we have considered an epidemic model with non monotonic incidence rate under the treatment.

Thus our model becomes

$$
\begin{aligned}
\frac{d S}{d t} & =a-d S-\frac{\lambda I S}{I+\alpha I^{2}}+\beta R, \\
\frac{d I}{d t} & =\frac{\lambda I S}{I+\alpha I^{2}}-(d+m) I-T(I), \\
\frac{d R}{d t} & =m I-(d+\beta) R+T(I),
\end{aligned}
$$

where $\mathrm{S}(\mathrm{t}), \mathrm{I}(\mathrm{t}), \mathrm{R}(\mathrm{t})$ denote the number of susceptible, infective, recovered individuals, respectively; a is the recruitment rate of the population, $\mathrm{d}$ is the natural death rate of the population, $\lambda$ is the proportionality constant, $\mathrm{m}$ is the natural recovery rate of the infective individuals, $\beta$ is the rate at which recovered individuals lose immunity and return to susceptible class, $\alpha$ is the parameter measures of the psychological or inhibitory effect. In our work we take the treatment function T (I), defined by

$$
\begin{aligned}
T(I) & =r I, \text { if } 0 \leq I \leq I_{0} \\
& =K_{1}, \text { if } I>I_{0},
\end{aligned}
$$

This means that the treatment rate is proportional to the infective when the number of infective is less or equal to some fixed value $I_{0}$ and the treatment is constant when the number of infective crosses the fixed value $I_{0}$. In practical view, the above form of treatment function is justified where patients have to be hospitalized and the number of beds is limited or the medicines are not sufficient.

Part I: SIR model with $0 \leq I \leq I_{0}$.

\section{Equilibrium states and their stability}

In this case the system (1)-(3) reduces to

$$
\begin{aligned}
\frac{d S}{d t} & =a-d S-\frac{\lambda I S}{I+\alpha I^{2}}+\beta R, \\
\frac{d I}{d t} & =\frac{\lambda I S}{I+\alpha I^{2}}-(d+m+r) I, \\
\frac{d R}{d t} & =(m+r) I-(d+\beta) R .
\end{aligned}
$$

The system of equations (6)-(8) always has the disease free equilibrium $E_{0}(a / d, 0,0)$ for any set of parameter values. The endemic equilibrium is the solution of

$$
\begin{aligned}
& a-d S-\frac{\lambda I S}{I+\alpha I^{2}}+\beta R=0, \\
& \frac{\lambda I S}{I+\alpha I^{2}}-(d+m+r) I=0, \\
& (m+r) I-(d+\beta) R=0 .
\end{aligned}
$$

From the third equations we get $R=\{(m+r) /(d+\beta)\} I$ and from the second equation $S=(d+m+r)\left(1+\alpha I^{2}\right) / \lambda$. Now substituting $\mathrm{R}$ and $\mathrm{S}$ in the first equation, we get

$$
\alpha d(d+m+r) I^{2}+\lambda\{d+m+r-\beta(m+r) /(d+\beta)\} I+d(d+m+r)-\lambda a=0 .
$$

We define the basic reproductive number as follows

$$
R_{0}=\frac{\lambda a}{d(d+m+r)}
$$

From the equation (9) we see that if $R_{0} \leq 1$, there is no positive solution as in that case coefficient of $I^{2}, I$ and constant term are all positive, but if $R_{0}>1$, then by Descartes rule there exists a unique positive solution of (9) and consequently there exists unique positive equilibrium $E^{*}\left(S^{*}, I^{*}, R^{*}\right)$, called endemic equilibrium. 
Here, $R^{*}=\{(m+r) /(d+\beta)\} I^{*}, S^{*}=(d+m+r)\left(1+\alpha I^{* 2}\right) / \lambda$ and

$$
I^{*}=\left[-K\{d+m+r-\beta(m+r) /(d+\beta)\}+\sqrt{\Delta_{1}}\right] /\{2 \alpha d(d+m+r)\},
$$

where $\Delta_{1}=\lambda^{2}\{d+m+r-\beta(m+r) /(d+\beta)\}^{2}-4 \alpha d^{2}(d+m+r)^{2}\left[1-R_{0}\right]$.

Obviously $\Delta_{1}>0$, when $R_{0}>1$.

To investigate the stability of the system, we first prove that $S(t)+I(t)+R(t)=a / d$ is invariant manifold of the system (6) - (8), which is attracting the first octant.

Let $N(t)=S(t)+I(t)+R(t)$, then

$\frac{d N}{d t}=a-d N(t)$, this imply $N(t)=A_{1} e^{-d t}+a / d$,

where $N\left(t_{0}\right)=A_{1} e^{-d t_{0}}+a / d$, therefore $N(t)=\left(N\left(t_{0}\right)-a / d\right) e^{-d\left(t-t_{0}\right)}+a / d$.

Thus $N(t) \rightarrow a / d$, as $t \rightarrow \infty$. So the limit set of system (6) - (8) is on the plane $S+I+R=a / d$.

Thus the reduced system is

$$
\begin{gathered}
\frac{d I}{d t}=\frac{\lambda I(a / d-I-R)}{1+\alpha I^{2}}-(d+m+r) I=F_{1}(I, R), \\
\frac{d R}{d t}=(m+r) I-(d+\beta) R=F_{2}(I, R) .
\end{gathered}
$$

Now to test the local stability of the above system we rescale the system by $x=\frac{\lambda I}{d+\beta}, y=\frac{\lambda R}{d+\beta}, T=(d+\beta) t$ and obtain

$$
\begin{gathered}
\frac{d x}{d T}=\frac{x(K-x-y)}{1+v x^{2}}-u x, \\
\frac{d y}{d T}=w x-y,
\end{gathered}
$$

where $K=\frac{a \lambda}{d(d+\beta)}, u=\frac{d+m+r}{d+\beta}, v=\frac{\alpha(d+\beta)^{2}}{\lambda^{2}}, w=\frac{m+r}{d+\beta}$.

Here $E_{0}(0,0)$ is the disease free equilibrium and the unique positive equilibrium $\left(x^{*}, y^{*}\right)$ of the system (14)-(15) is the endemic equilibrium $E^{*}$ of the model (6)-(8). ( $\left(x^{*}, y^{*}\right)$ exists if $u-K<0$ and is given by $u v x^{* 2}+(1+w) x^{*}+(u-K)=$ $0 ; y^{*}=w x^{*}$.

Therefore,

$$
x^{*}=\frac{-(1+w)+\sqrt{(1+w)^{2}-4 u v(u-K)}}{2 u v}, y^{*}=w x^{*} .
$$

The jacobian matrix corresponding to $E_{0}(0,0)$ is

$M_{0}=\left[\begin{array}{cc}K-u & 0 \\ w & -1\end{array}\right]$

Obviously (i) if $(K-u)>0,(0,0)$ is an unstable saddle point;

(ii) if $K=u,(0,0)$ is saddle node;

(iii) if $(K-u)<0,(0,0)$ is a stable node.

Here $(K-u)>0 \Leftrightarrow R_{0}>1$ and $(K-u)<0 \Leftrightarrow R_{0}<1$.

So, whenever $E^{*}$ exists, $E_{0}$ turns to an unstable saddle point.

Now when $(K-u)>0$ i.e. $R_{0}>1$, we discuss the stability of endemic equilibrium $\left(x^{*}, y^{*}\right)$.

Jacobian matrix corresponding to $\left(x^{*}, y^{*}\right)$ is

$M_{1}=\left[\begin{array}{cc}x^{*}\left(v x^{* 2}+2 v w x^{* 2}-2 K v x^{*}-1\right) /\left(1+v x^{* 2}\right)^{2} & -x^{*} /\left(1+v x^{* 2}\right) \\ w & -1\end{array}\right]$.

The sign of $\operatorname{det}\left(M_{1}\right)=x^{*}\left\{1+w+2 K v x^{*}-v(1+w) x^{* 2}\right\} /\left(1+v x^{* 2}\right)^{2}$ is determined by the sign of

$$
P_{1}=-v(1+w) x^{* 2}+2 K v x^{*}+(1+w) .
$$




$$
\text { We have } u v x^{* 2}+(1+w) x^{*}+(u-K)=0 \text {. }
$$

Now $u \times(18)+(1+w) \times(17) \Rightarrow u P_{1}=\left\{2 K u v+(1+w)^{2}\right\}\left\{x^{*}+\frac{(1+w)(2 u-K)}{2 K u v+(1+w)^{2}}\right\}$.

Now substituting $x^{*}=\left\{-(1+w)+\Delta_{1}\right\} / 2 u v$, where $\Delta_{1}=\sqrt{(1+w)^{2}-4 u v(u-K)}$, we get

$u P_{1}=\frac{1}{2 u v}\left[-(1+w) \Delta_{1}^{2}+\Delta_{1}\left\{2 K u v+(1+w)^{2}\right\}\right]=\frac{-\Delta_{1}}{2 u v}\left[(1+w) \Delta_{1}-\left\{2 K u v+(1+w)^{2}\right\}\right]$.

$\therefore P_{1}=\frac{-\Delta_{1}}{2 u^{2} v}\left[(1+w) \Delta_{1}-\left\{2 K u v+(1+w)^{2}\right\}\right]=\frac{(1+w) \Delta_{1}}{2 u^{2} v}\left[\left(1+w+\frac{2 K u v}{1+w}\right)-\Delta_{1}\right]$.

Since

$\left(1+w+\frac{2 K u v}{1+w}\right)^{2}-\Delta_{1}^{2}=\frac{4 K^{2} u^{2} v^{2}}{(1+w)^{2}}+4 u^{2} v>0$,

therefore $P_{1}>0$, and hence det $\left(M_{1}\right)$ is positive for any set of parameters.

Therefore, the positive equilibrium $\left(x^{*}, y^{*}\right)$ is either a node, a focus or a center. The eigen values of $M_{1}$ are $\lambda=$ $\frac{1}{2}\left(\operatorname{Trace}\left(M_{1}\right) \pm \sqrt{\left(\operatorname{Trace}\left(M_{1}\right)\right)^{2}-4 \operatorname{det}\left(M_{1}\right)}\right)$.

The fact that $\operatorname{det}\left(M_{1}\right)>0$ implies that

$\left|\operatorname{Trace}\left(M_{1}\right)\right|>\sqrt{\left(\operatorname{Trace}\left(M_{1}\right)\right)^{2}-4 \operatorname{det}\left(M_{1}\right)}$.

The stability of the $\left(x^{*}, y^{*}\right)$ depends on the sign of the Trace and determinant of the jacobian matrix:

$\left(x^{*}, y^{*}\right)$ is stable if Trace $\left(M_{1}\right)<0$, unstable if Trace $\left(M_{1}\right)>0 .\left(x^{*}, y^{*}\right)$ is a node if $\left(\operatorname{Trace}\left(M_{1}\right)\right)^{2}>4 \operatorname{det}\left(M_{1}\right)$ and a focus if $\left(\operatorname{Trace}\left(M_{1}\right)\right)^{2}<4 \operatorname{det}\left(M_{1}\right)$.

Now Trace $\left(M_{1}\right)=\left\{-v^{2} x^{* 4}+(1+2 w) v x^{* 3}-2(1+K) v x^{* 2}-x^{*}-1\right\} /\left(1+v x^{* 2}\right)^{2}$.

So the sign of Trace $\left(M_{1}\right)$ is determined by

$$
P_{2}=-v^{2} x^{* 4}+(1+2 w) v x^{* 3}-2(1+K) v x^{* 2}-x^{*}-1 .
$$

After some algebraic calculation using (18) and (19) we get, $u^{3} v P_{2}=P_{3} x^{*}-P_{4}$, where

$P_{3}=(1+w)\left[(1+w)^{2}+u(1+w)(1+2 w)+2 u v K\right]+u^{2} v K(1+2 w)+2 u^{2} v(1+w)(K-u)$,

$P_{4}=(K-u)\left[(1+w)^{2}+u(1+w)(1+2 w)+u v\left[(K-u)^{2}+2 u(K-u)(1+K)+u^{2}\right]\right.$.

Therefore $P_{3}$ and $P_{4}$ are positive for any set of parameters with $K>u$. So when $\left(x^{*}, y^{*}\right)$ exists, the condition for the local stability of $\left(x^{*}, y^{*}\right)$ becomes $x^{*}<P_{4} / P_{3}$.

The above discussion can be stated through a theorem.

Theorem 2.1. (i) When the basic reproductive number $R_{0} \leq 1$, there exist no positive equilibrium of the system (14) (15), and in that case the only disease free equilibrium $(0,0)$ is a stable node.

(ii) When $R_{0}>1$, there exists a unique positive equilibrium of the system (14) - (15), and in that case $(0,0)$ is an unstable saddle point. Also the condition for which the unique positive equilibrium will be locally stable is $x^{*}<P_{4} / P_{3}$.

Global Stability. To investigate the global stability of the disease free equilibrium it is sufficient to show that $(I(t), R(t)) \rightarrow$ $(0,0)$. From here, it is clear that $S(t) \rightarrow a / d$. Now from positivity of the solutions, $I(t)$ and $R(t)$ satisfy the differential inequality given by

$$
\begin{aligned}
& \frac{d I}{d t} \leq\left\{\frac{\lambda a}{d}-(d+m+r)\right\} I=\frac{d i}{d t}, \\
& \frac{d R}{d t} \leq(m+r) I-(d-\beta) R=\frac{d r}{d t} .
\end{aligned}
$$

Here $i(t), r(t)$ are linear, and $(i(t), r(t)) \rightarrow(0,0)$ as $t \rightarrow \infty$ if $\lambda \frac{a}{d}-(d+m+r)<0$ i.e. $R_{0}<1$.

Since $I(t) \leq i(t)$ and $R(t) \leq r(t),(I(t), R(t)) \rightarrow(0,0)$ as $t \rightarrow \infty$ by simple comparison argument. Hence disease free equilibrium is globally stable.

Now to investigate whether system (12) - (13) admits limit cycle or not, we take Dulac function $D(I, R)=\left(1+\alpha I^{2}\right) / \lambda I$, then 
$\partial\left(D F_{1}\right) / \partial I+\partial\left(D F_{2}\right) / \partial R=-1-\{2 \alpha(d+m+r) / \lambda\} I-\left\{(d+\beta)\left(1+\alpha I^{2}\right)\right\} / \lambda I<0$,

hence the system (12)-(13) has no limit cycle in the positive quadrant, so we reach the theorem 2.2.

Theorem 2.2. If $R_{0}<1$, then the disease free equilibrium $E_{0}(a / d, 0,0)$ of the system (12) - (13) is globally stable. But when $R_{0}>1$, system (12)-(13) have unique positive equilibrium and further when $x^{*}<P_{4} / P_{3}$ that unique positive equilibrium must be locally stable. Again since the system have no limit cycle in the positive quadrant, $E^{*}\left(x^{*}, y^{*}\right)$ must be globally stable under the condition $R_{0}>1$ and $x^{*}<P_{4} / P_{3}$.

Part II. SIR model with $I>I_{0}$.

\section{Equilibrium states and their stability}

In this case the model reduces to

$$
\begin{gathered}
\frac{d S}{d t}=a-d S-\frac{\lambda I S}{1+\alpha I^{2}}+\beta R, \\
\frac{d I}{d t}=\frac{\lambda I S}{1+\alpha I^{2}}-(d+m) I-K_{1}, \\
\frac{d R}{d t}=m I-(d+\beta) R+K_{1} .
\end{gathered}
$$

Since $S+I+R=a / d$ is invariant manifold of the system (22)-(24), the model reduces to

$$
\begin{gathered}
\frac{d I}{d t}=\frac{\lambda I(a / d-I-R)}{1+\alpha I^{2}}-(d+m) I-K_{1}, \\
\frac{d R}{d t}=m I-(d+\beta) R+K_{1} .
\end{gathered}
$$

Substituting $\quad x=\frac{\lambda I}{d+\beta}, y=\frac{\lambda R}{d+\beta}, T=(d+\beta) t$

we get

$$
\begin{gathered}
\frac{d x}{d T}=\frac{x(L-x-y)}{\left(1+v_{1} x^{2}\right)}(L-x-y)-u_{1} x-c, \\
\frac{d y}{d T}=w_{1} x-y+c,
\end{gathered}
$$

where $v_{1}=v=\alpha(d+\beta)^{2} / \lambda^{2}, L=K=a \lambda /\{d(d+\beta)\}, u_{1}=(d+m) /(d+\beta)$, $c=\lambda K_{1} /(d+\beta)^{2}, w_{1}=m /(d+\beta)$.

For equilibrium $x(L-x-y)-u_{1} x\left(1+v_{1} x^{2}\right)-c\left(1+v_{1} x^{2}\right)=0$,

or,

$$
u_{1} v x^{3}+\left(1+w_{1}+c v\right) x^{2}+\left(c+u_{1}-K\right) x+c=0
$$

If $u_{1}+c>K$, (29) has no positive solution, but if $u_{1}+c<K$, it has either two positive roots or no positive root. By theory of equation

$$
a_{0} x^{3}+3 a_{1} x^{2}+3 a_{2} x+a_{3}=0
$$

has all of its roots real if $G^{2}+4 H^{3}<0$ and $H<0$, where $H=a_{0} a_{2}-a_{1}^{2}$,

$G=a_{0}^{2} a_{3}-3 a_{0} a_{1} a_{2}+2 a_{1}^{3}$. Comparing equation (29) to (30) we have $a_{0}=u_{1} v$,

$a_{1}=\left(1+w_{1}+c v\right) / 3, a_{2}=\left(u_{1}+c-K\right) / 3$, and $a_{3}=c$.

Here $H=a_{0} a_{2}-a_{1}^{2}=u_{1} v\left\{\left(u_{1}+c-K\right) / 3\right\}-\left\{\left(1+w_{1}+c v\right) / 3\right\}^{2}<0$, for $u_{1}+c<K$.

$G^{2}+4 H^{3}=\left(a_{0}^{2} a_{3}-3 a_{0} a_{1} a_{2}+2 a_{1}^{3}\right)^{2}+4\left(a_{0} a_{2}-a_{1}^{2}\right)^{3}$

$=a_{0}^{2}\left(a_{0}^{2} a_{3}^{2}-6 a_{0} a_{1} a_{2} a_{3}+4 a_{3} a_{1}^{3}+4 a_{0} a_{2}^{3}-3 a_{1}^{2} a_{2}^{2}\right)$.

Therefore

$$
G^{2}+4 H^{3}<0, \text { if }\left(a_{0}^{2} a_{3}^{2}+4 a_{3} a_{1}^{3}+4 a_{0} a_{2}^{3}\right)<\left(3 a_{1}^{2} a_{2}^{2}+6 a_{0} a_{1} a_{2} a_{3}\right)
$$

To investigate the local stability of the positive equilibrium $(\bar{x}, \bar{y})$ of the system (27)-(28), we consider the jacobian matrix $M_{2}(\bar{x}, \bar{y})=\left[\begin{array}{cc}\frac{\left\{\left(1+v \bar{x}^{2}\right)(K-\bar{x}-\bar{y}-\bar{x})-2 v \bar{x}\left(k \bar{x}-\bar{x}^{2}-\bar{x} \bar{y}\right)\right.}{1+v \bar{x}^{2}}-u_{1} & \frac{-\bar{x}}{1+v \bar{x}^{2}} \\ w_{1} & -1\end{array}\right]$.

Now

$\operatorname{det}\left(M_{2}\right)$ 
$=\frac{-\left(v \bar{x}^{2}+1\right)\left(K-2 \bar{x}-w_{1} \bar{x}-c\right)+2 v \bar{x}\left(K \bar{x}-\bar{x}^{2}-w_{1} \bar{x}^{2}-c \bar{x}\right)+u_{1}\left(v \bar{x}^{2}+1\right)^{2}+w_{1} \bar{x}\left(v \bar{x}^{2}+1\right)}{\left(1+v \bar{x}^{2}\right)^{2}}$

Sign of $\operatorname{det}\left(M_{2}\right)$ is determined by

$$
P_{5}=u_{1} v^{2} \bar{x}^{4}+\left(K v-v c+2 u_{1} v\right) \bar{x}^{2}+\left(2+2 w_{1}\right) \bar{x}+\left(c+u_{1}-K\right) .
$$

Now $(32)-v \bar{x} \times(29) \Rightarrow$

$$
P_{5}=-v\left(1+w_{1}+c v\right) \bar{x}^{3}+v\left(2 K+u_{1}-2 c\right) \bar{x}^{2}+\left(2+2 w_{1}-v c\right) \bar{x}+\left(c+u_{1}-K\right) .
$$

Again $\left(1+w_{1}+c v\right) \times(29)+u_{1} \times(33) \Rightarrow$

$u_{1} P_{5}=\bar{x}^{2}\left\{2 K u_{1} v+u_{1}^{2} v-2 u_{1} v c+\left(1+w_{1}+c v\right)^{2}\right\}+\bar{x}\left\{\left(2 u_{1}+2 u_{1} w_{1}-u_{1} v c\right)+\right.$

$\left.\left(1+w_{1}+c v\right)\left(u_{1}+c-K\right)\right\}+\left\{\left(c u_{1}+u_{1}^{2}-K u_{1}\right)+c\left(1+w_{1}+c v\right)\right\}$.

$\therefore u_{1} P_{5}=\xi_{1} \bar{x}^{2}+\xi_{2} \bar{x}+\xi_{3}$, where $\xi_{1}=\left\{u_{1}^{2} v+\left(1+w_{1}+c v\right)^{2}+2 u_{1} v(K-c)\right\}>0$ for $K>c$.

So, the sufficient condition for which $P_{5}>0$ is

$$
\xi_{2}^{2}-4 \xi_{1} \xi_{2} \leq 0
$$

Now Trace $\left(M_{2}\right)=\frac{\left\{\left(1+v \bar{x}^{2}\right)(K-2 \bar{x}-\bar{y})-2 v \bar{x}^{2}(K-\bar{x}-\bar{y})\right\}}{\left(1+v \bar{x}^{2}\right)^{2}}-\left(u_{1}+1\right)$

$=\frac{\left\{\left(1+v \bar{x}^{2}\right)\left(K-2 \bar{x}-w_{1} \bar{x}-c\right)-2 v \bar{x}^{2}\left(K-\bar{x}-w_{1} \bar{x}-c\right)-\left(u_{1}+1\right)\left(1+v \bar{x}^{2}\right)^{2}\right\}}{\left(1+v \bar{x}^{2}\right)^{2}}$.

So the sign of Trace $\left(M_{2}\right)$ is determined by

$$
P_{6}=-\left(u_{1}+1\right) v^{2} \bar{x}^{4}+v w_{1} \bar{x}^{3}+\left(v c-K v-2 v u_{1}-2 v\right) \bar{x}^{2}-\left(2+w_{1}\right) \bar{x}+\left(K-c-u_{1}-1\right) .
$$

After some algebraic calculation using (29) and (35) we get

$u_{1}^{2} P_{6}=\eta_{1} \bar{x}^{2}+\eta_{2} \bar{x}+\eta_{3}$,

where

$\eta_{1}=-\left[\left(1+w_{1}+c v\right)\left(2 u_{1} w_{1}+u_{1} c v+u_{1}+w_{1}+c v+1\right)+u_{1} v\left\{(K-c)\left(1+2 u_{1}\right)+u_{1}^{2}+u_{1}\right\}\right]<0$,

for $K>u_{1}+c$,

$\eta_{2}=u_{1}\left(c v u_{1}+c v-2 u_{1}-w_{1} u_{1}\right)-\left(u_{1}+c-K\right)\left(2 u_{1} w_{1}+u_{1} c v+u_{1}+w_{1}+c v+1\right)$,

$\eta_{3}=u_{1}\left(K-c-u_{1}-1\right)-c\left(2 u_{1} w_{1}+u_{1} c v+u_{1}+w_{1}+c v+1\right)$.

Therefore the sufficient condition for which $P_{6}<0$ is

$$
\eta_{2}^{2}-4 \eta_{1} \eta_{3} \leq 0
$$

So we reach the theorem 3.1

Theorem 3.1. When $K>u_{1}+c$, the system (27)-(28) has two positive equilibrium $\left(\bar{x}_{1}, \bar{y}_{1}\right)$ and $\left(\bar{x}_{2}, \bar{y}_{2}\right)$, where $\bar{x}_{1}, \bar{x}_{2}$ are two positive solutions of the equation (29) under the parametric restriction given by (31), moreover when the conditions (34) and (36) are satisfied at some equilibrium point, that equilibrium point must be asymptotically stable.

\section{Simulation and Discussion}

Case I. $0 \leq I \leq I_{0}$ : If we choose the parameters as follows:

$a=3, d=0.1, \lambda=0.3, \alpha=0.5, \beta=0.1, m=0.01, r=0.2$, then we get the unique positive equilibrium point (18.18827, $5.76243,6.050551)$. Here the basic reproductive number $R_{0}=29.03226>1$. For the above choice of parameters we see that all the three components $S(t), I(t), R(t)$ approach to their steady state values as time goes to infinity, the disease becomes endemic (see figure 2).

$<$ Figure $1>$

Again if we take $a=15, d=2.5, \lambda=0.5, \alpha=1, \beta=0.5, m=10$ and $r=0.1$, the value of the basic reproductive number becomes $0.2380952<1$ and in that case we see that, the disease dies out (see figure 3 ).

$<$ Figure 2-3>

By rescaling, the system (14) \& (15) reduces to

$\frac{d x}{d T}=\frac{x(45-x-y)}{1+0.2222222 x^{2}}-1.55 x$

$\frac{d y}{d T}=1.05-y$.

Here $(u-K)<0$, and hence there exists unique positive equilibrium point $\left(x^{*}, y^{*}\right)$ where $x^{*}=9.075827$ and $y^{*}=8.643645$. For the above choice of parameters $P_{3}=9.162602>0 P_{4}=196.5196, P_{4} / P_{3}=21.44801$ and therefore the sufficient 
condition for local stability i.e. $x^{*}<P_{4} / P_{3}$ is satisfied here. We have drawn figures for both the system $(S(t), I(t), R(t)$ and $(x(T), y(T))$ to verify our result (see figures 4 and 5 ).

$<$ Figure 4-5 >

Figures 4 and 5 also shows that there exists no limit cycle and the unique positive equilibrium (18.18827, 5.76243, $6.050551)$ of the system (6)-(8) or equivalently $(9.075827,8.643645)$ of the system (14)-(15) is globally stable.

In our model parameter $\alpha$ describes the psychological or inhibitory effect. From (11), we see that the steady state value $I^{*}$ of the infective decreases as $\alpha$ increases. To verify the result we have plotted figure 6 for different values of $\alpha$, keeping all other values of the parameters same as for figure 2 .

$<$ Figure 6-7 >

We have also plotted figure 7 for different values of $\mathrm{m}$, keeping all others parameter values same as for figure 2 and see that the steady state value $I^{*}$ decreases as $m$ increases. Further we have plotted figure 8 to see the dependence of $I^{*}$ on the parameter $\mathrm{r}$ and see that $I^{*}$ decreases as $\mathrm{r}$ increases.

$<$ Figure 8-9>

To see the dependence of the steady state value $S^{*}$ of the susceptible on the parameter $\mathrm{r}$, we have plotted figure10 and have seen that $S^{*}$ decreases as the parameter $\mathrm{r}$ increases, keeping all other parameters same. $<$ Figure $10>$

Case (II): $I>I_{0}$ To study the system (1)-(3) numerically, where $I>I_{0}$, we choose our parameters as, $a=2.8, d=$ $0.0453, \lambda=0.4, \alpha=2.0, \beta=0.13, m=0.01, K_{1}=0.7$.

Here $S+I+R=(a / d)=61.810154$ is invariant manifold. So the system reduces to $d I / d t=\left\{(0.4) I /\left(1+2 I^{2}\right)\right\}(61.810154-$ $I-R)-(0.0553) I-0.7$,

$d R / d t=(0.01) I-(0.1753) R+0.7$.

To rescale the system we consider

$x=\{\lambda /(d+\beta)\}$ I i.e. $x=(2.2818026) I, y=\{\lambda /(d+\beta)\}$ R i.e. $y=(2.2818026) R, T=(0.1753) t$, which reduces the above system to $d x / d T=\left\{x(141.2386-x-y) /\left(1+0.3841261 x^{2}\right)\right\}-(0.3154592) x-9.111591, d y / d T=(0.5704507) x-y+$ 9.111591 .

Now to find the equilibrium point of the above system we see that $u_{1}+c=9.4270502<141.0386, K$ and hence equation (29) has either two positive roots or no positive root. But here $a_{0}=0.1211761, a_{1}=1.519015, a_{2}=-43.87051$, and $a_{3}=$ $c=9.111591$. So,$H=-7.623465<0$, and

$\left(a_{0}^{2} a_{3}^{2}+4 a_{3} a_{1}^{3}+4 a_{0} a_{2}^{3}\right)-\left(3 a_{1}^{2} a_{2}^{2}+6 a_{0} a_{1} a_{2} a_{3}\right)=-53721.47<0$, i.e.

is satisfied which imply that $G^{2}+4 H^{3}<0$. Therefore for our choice of parameters the system (27)-(28) has two positive equilibrium $(19.0878,10.2004)$ and $(0.0694,9.1155)$.

$<$ Figure $11>$

Now at $(19.0878,10.2004)$ the value of the $\operatorname{det}\left(M_{2}\right)=24659.46>0$ and Trace $\left(M_{2}\right)=-42992.9<0$, therefore $(19.0878$, 10.2004) will be asymptotically stable.

Figure 13 shows that $(19.0878,10.2004)$ is a stable node, also figure 12 shows that the corresponding equilibrium point $(48.96,8.36,4.47)$ of the system (22)-(24) is a stable node.

\section{$<$ Figure 12-13 >}

Again at the other equilibrium point $(0.694,9.1155)$, value of Det $\left(M_{2}\right)=-131.1483<0$ and $\operatorname{Trace}\left(M_{2}\right)=130.1843>0$ and hence it becomes unstable.

Figure 14 shows the dependence of the steady state value $I^{*}$ of $\mathrm{I}(\mathrm{t})$ on the parameter $K_{1}$ and we see that $I^{*}$ decreases as $K_{1}$ increases.

\section{$<$ Figure 14-16 >}

We see that basic reproductive number plays an important role to control the disease. When $R_{0} \leq 1$, there exists no positive equilibrium, and in that case the disease free equilibrium is globally stable, that is the disease dies out. But when $R_{0}>1$, the unique endemic equilibrium is globally stable under some parametric condition. Also we see that the treatment rate plays a major role to control the disease. From figure $14(\mathrm{~b})$, we can see that when the value of the parameter $K_{1}$ crosses a definite value 1.27, the disease dies out. Figure 15 and 16 show that number of susceptible and recovered increases as the value of the parameter $K_{1}$ increases.

\section{References}

Alexander M. E. and Moghadas S. M. (2004). Periodicity in an epidemic model with a generalized non-linear incidence. Math. Biosci., 189, 75-96. 
Anderson R.M. and May R.N. (1979). population biology of infectious disease I. Nature 180, 316-367.

Derrick W. R. and Van den Driessche P. (1993). A disease transmission model in a non constant population. J. Math. Biol., 31, 495-512.

Esteva L. and Matias M. (2001). A model for vector transmitted diseases with saturation incidence. Journal of Biological Systems, 9, (4) 235-245.

Feng Z.and Thieme H. R. (1995). Recurrent out break of childhood diseases revisited: The impact of isolution. Math. Biosci., 128, 93.

Hethcote H.W. and Van den Driessche P. (1991). Some epidemiological models with non-linear incidence. J. math. Boil., 29, 271-287.

Hethcote H.W. and Levin S. A. (1988). periodicity in epidemiological models. In mathematical Ecology, volume II, ed. By Levin S.A., Hallam T.G. and Gross L.: springer verlag, New york.

Hyman J. M. and Li J. (1998). Modelling the effectiveness of isolation strategies in preventing STD epidemics. SIAM J. Appl. Math. 58, 912.

Kermack W.O. and Mckendrick A.G. (1927). A contribution to the mathematical theory of epidemic. Proc. R. Soc., London A115,700-721.

Liu W. M., Hethcote H.W. and Levin S. A. (1987). Dynamical behavior of epidemiological models with nonlinear incidence rates. J. Math. Biol., 25, 359-380.

Liu W. M., Levin S.A. and Iwasa Y. (1986). Influence of nonlinear incidence rates upon the behavior of SIRS epidemiological models. J. Math. Biol., 187-204.

Ruan S. and Wang W. (2003). Dynamical behavior of an epidemic model with nonlinear incidence rate. J. Differential equations, 188, 135-163.

Wang W. (2006). Backward bifurcation of an epidemic model with treatment. Math. Biosci., 201, 58-71.

Wu L., Feng Z. (2000). Homoclinic bifurcation in an SIQR model for childhood disesses. J. Differ. Equat., $168,150$.

Xiao D. and Ruan S.(2007). Global analysis of an epidemic model with non monotone incidence rate. Math. Biosci. 208, 419-429.

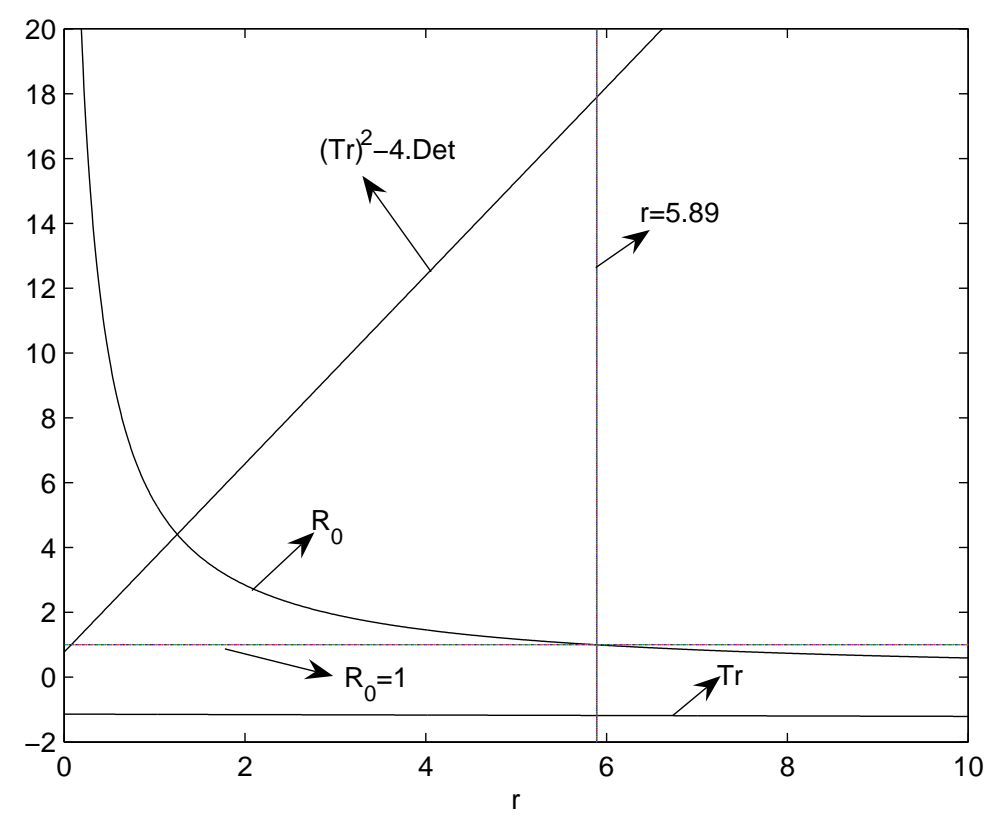

Figure 1. Bifurcation diagram for endemic and disease free equilibrium 


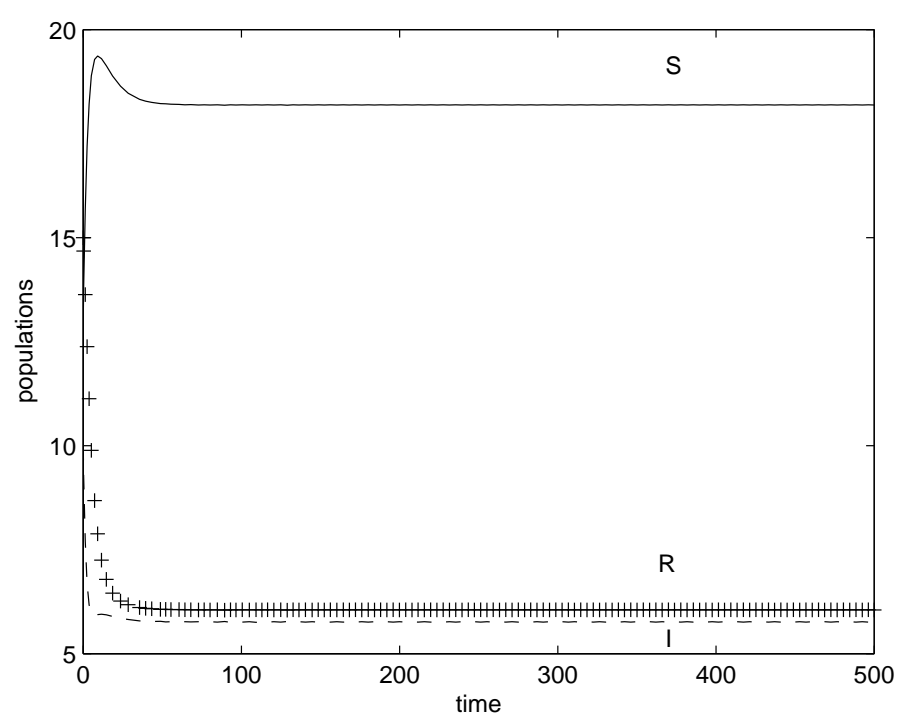

Figure 2. The plot represents that the SIR epidemic model is asymptotically stable. This plot is the numerical result of the stability analysis

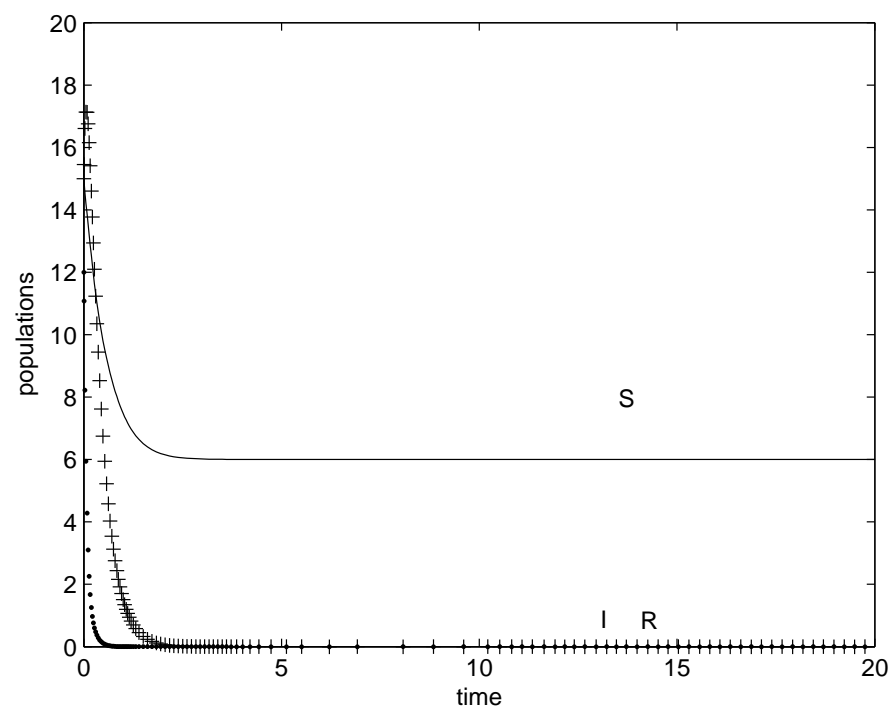

Figure 3. The plot represents that the disease dies

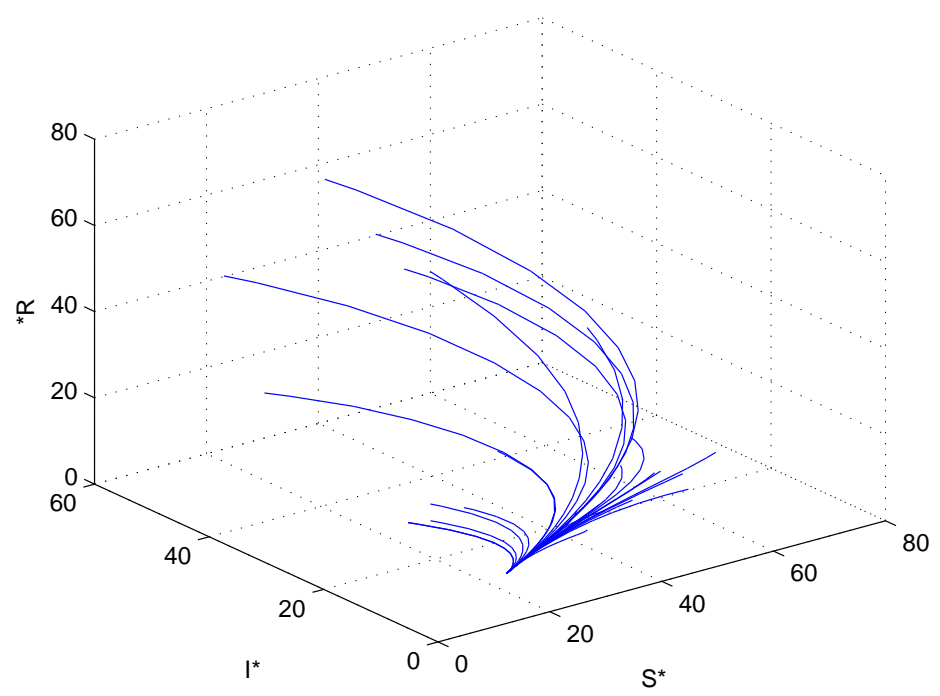

Figure 4. Equilibrium point $(18.18827,5.76243,6.0550551)$ of the system (6)-(8) globally asymptotically stable 


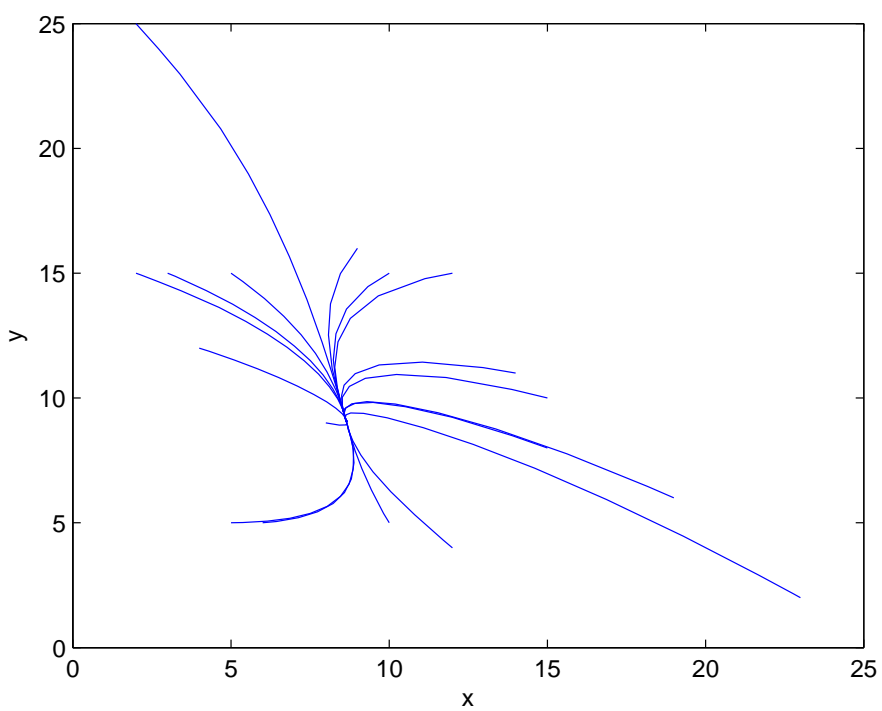

Figure 5. Equilibrium point $(9.075827,8.643645)$ of the system (14)-(15) is globally stable

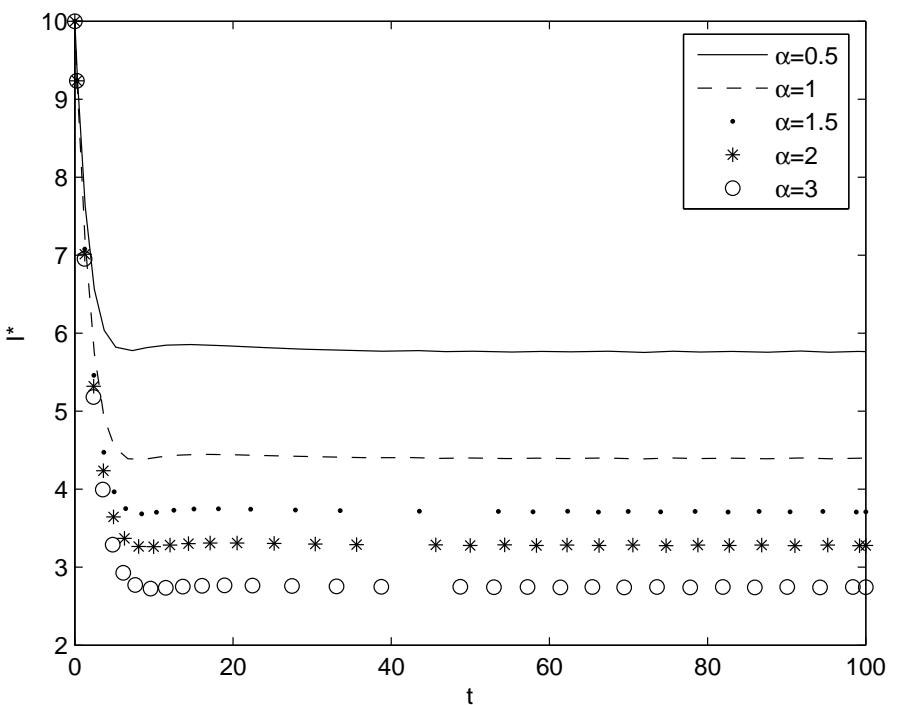

Figure 6. This figure shows the dependence of $I^{*}$ on the parameter $\alpha$

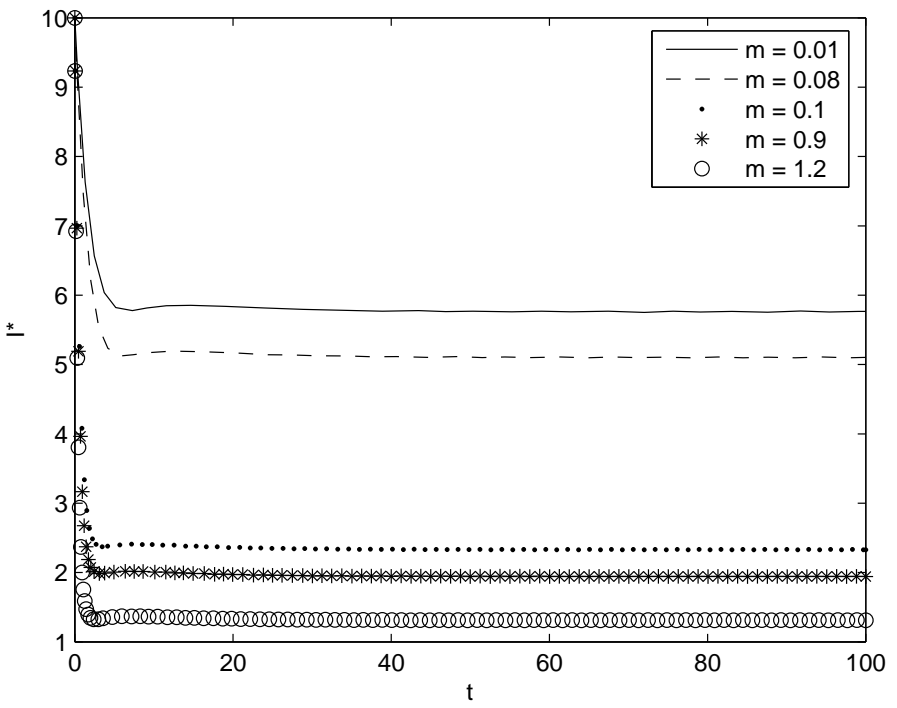

Figure 7. This figure shows the dependence of $I^{*}$ on the parameter $\mathrm{m}$ 


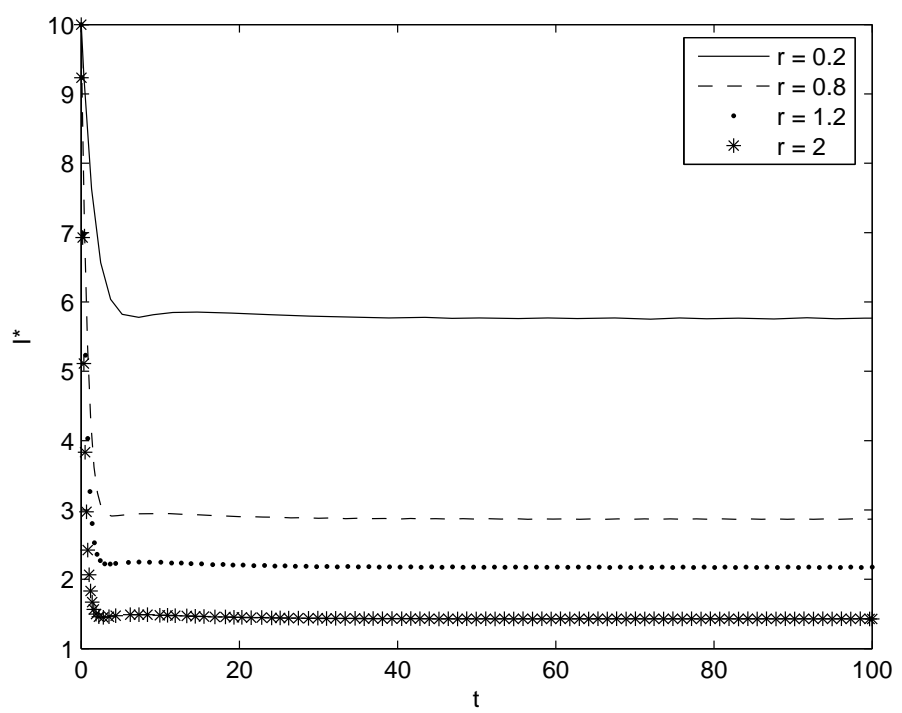

Figure 8. This figure shows the dependence of $I^{*}$ on the parameter $\mathrm{r}$

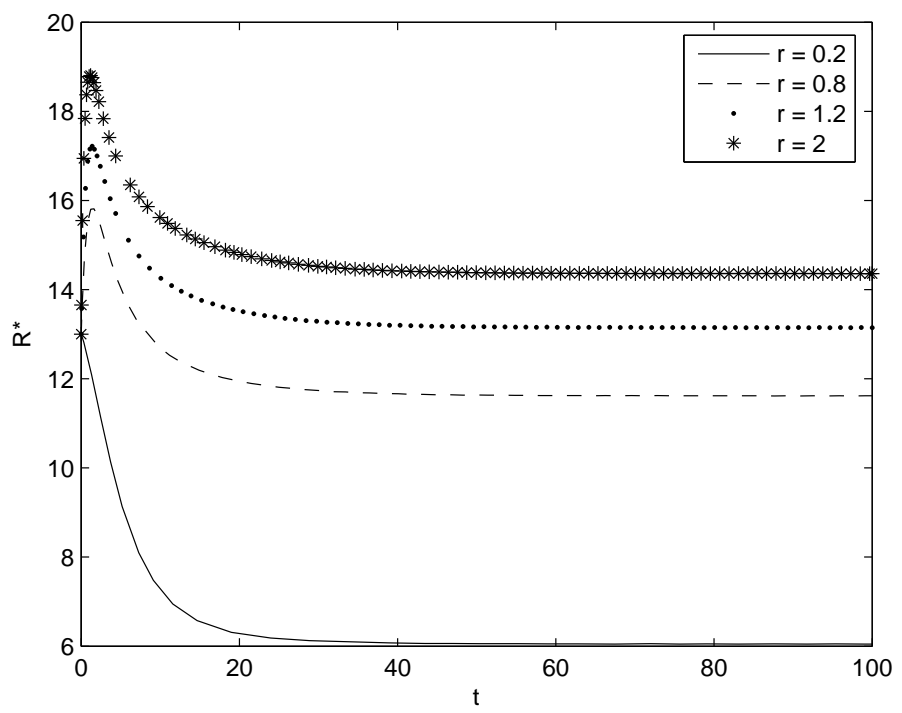

Figure 9. This figure shows the dependence of $R^{*}$ on the parameter $\mathrm{r}$

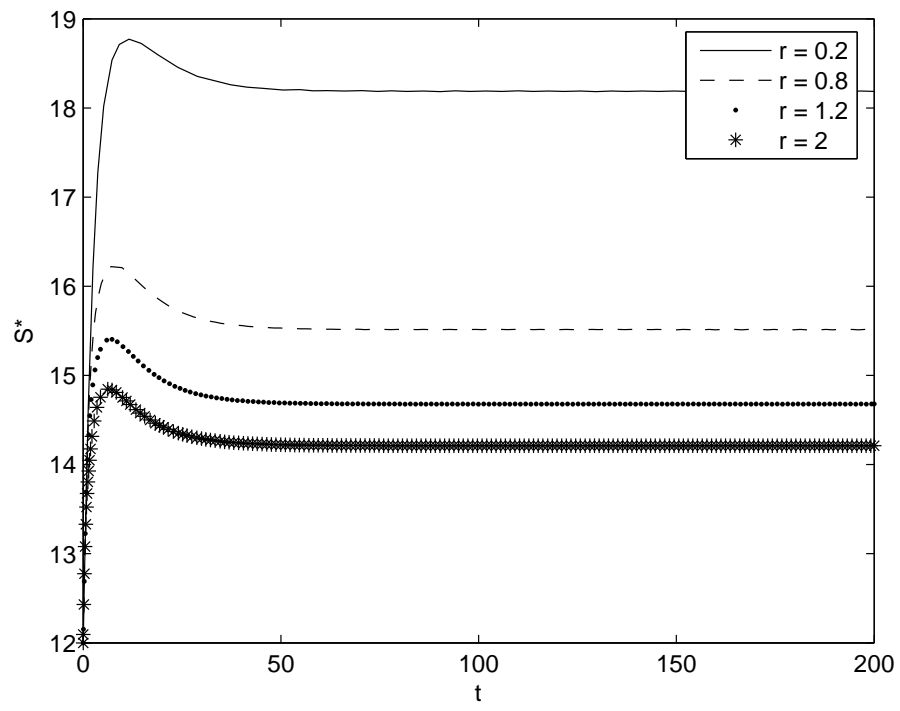

Figure 10. This figure shows the dependence of $S^{*}$ on the parameter $\mathrm{r}$ 


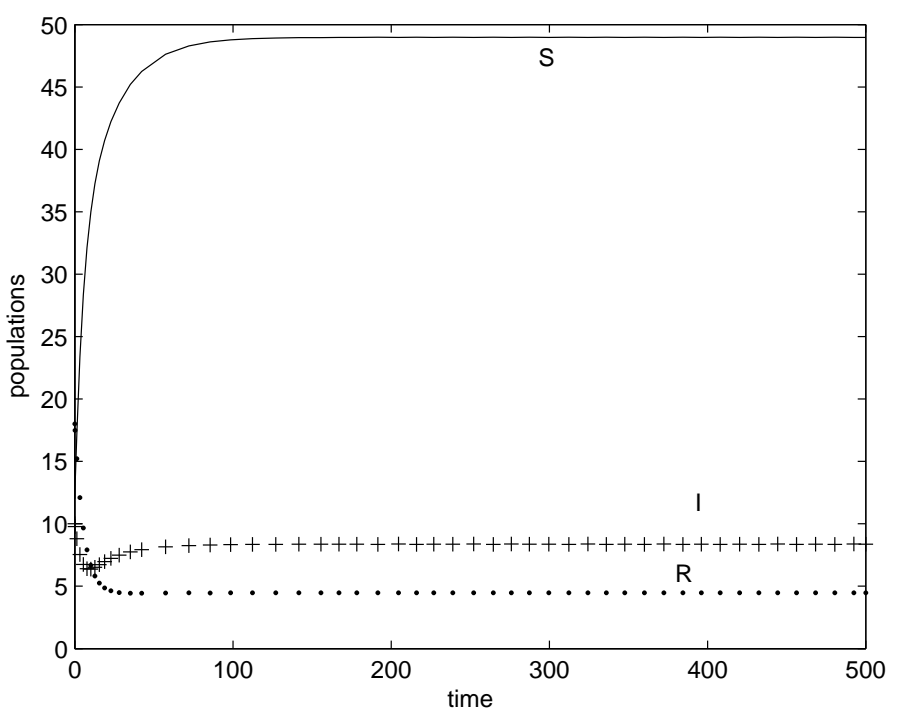

Figure 11. This figure shows that the populations approach their steady state as time goes to infinity and the disease become endemic

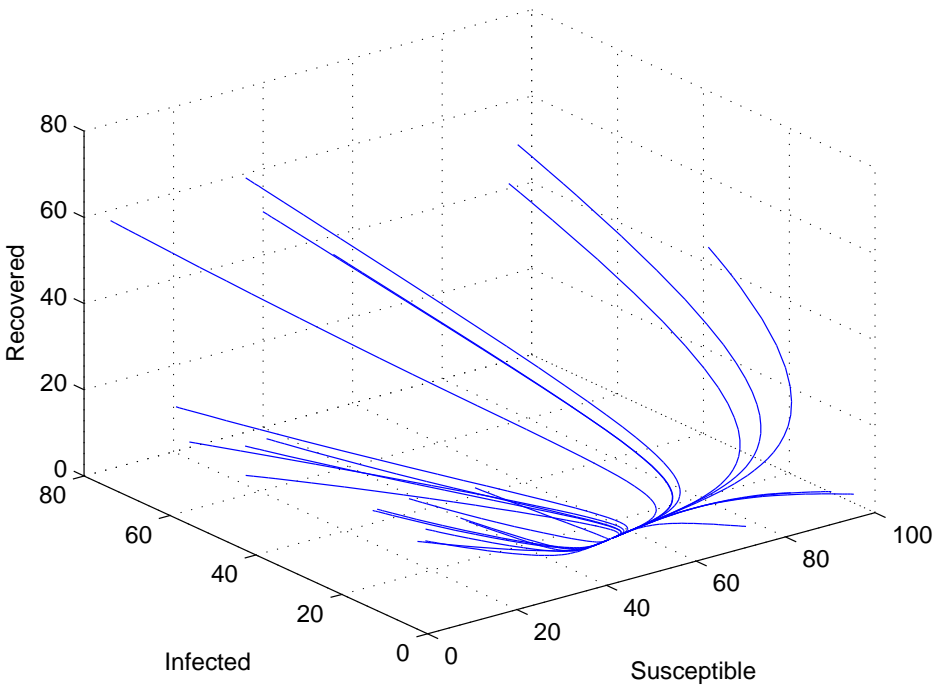

Figure 12. Equilibrium point $(48.96,8.36,4.47)$ of the system (22)-(24) is globally asymptotically stable

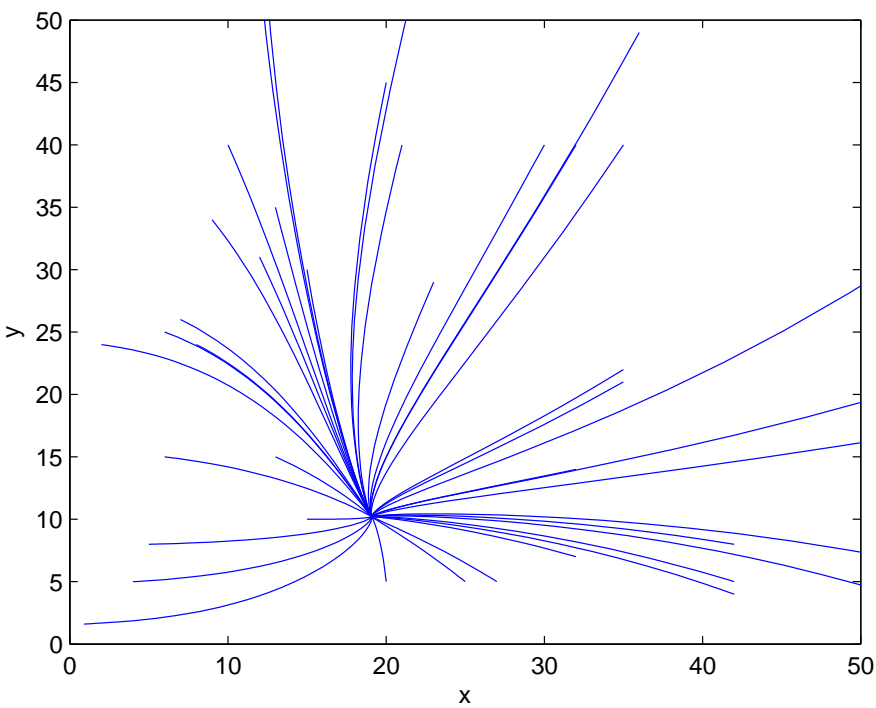

Figure 13. Equilibrium point $(19.0878,10.2004)$ of the system $(27)-(28)$ is globally stable 


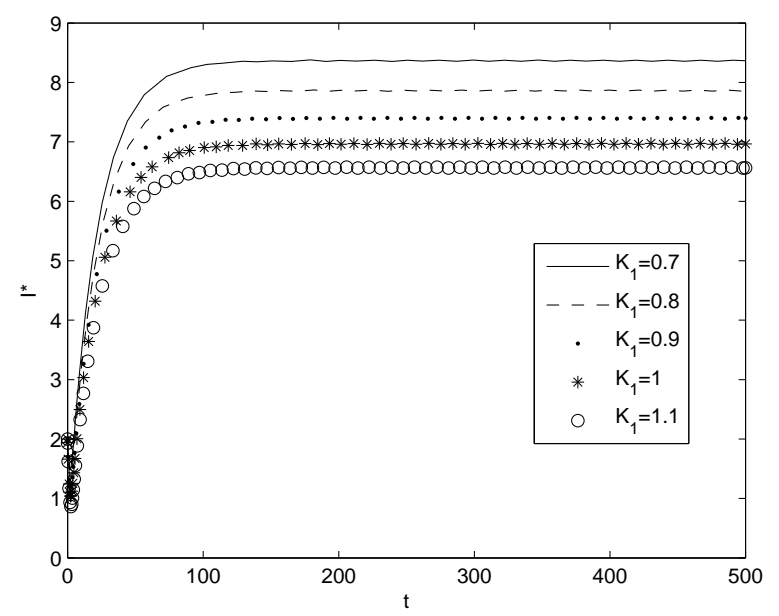

(14a)

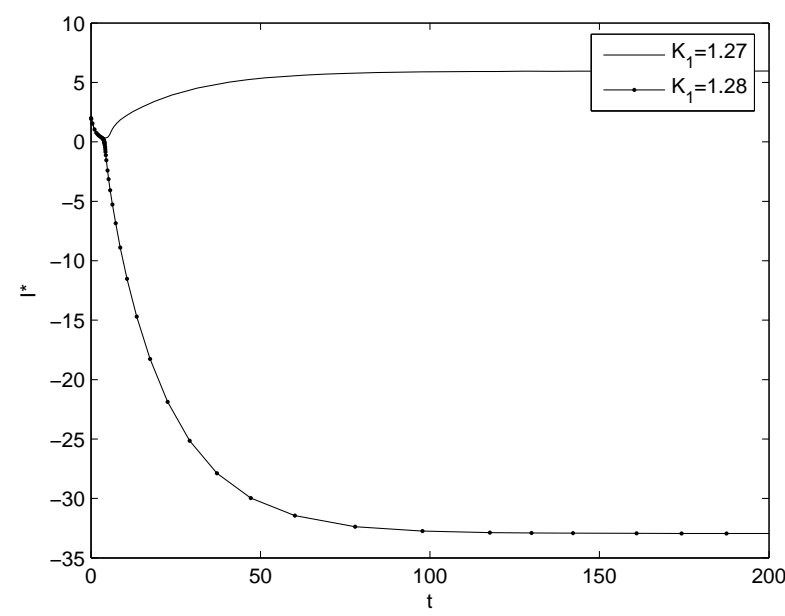

(14b)

Figure 14. $14 \mathrm{a}$ and $14 \mathrm{~b}$ show the dependence of $I^{*}$ on the parameter $K_{1}$.

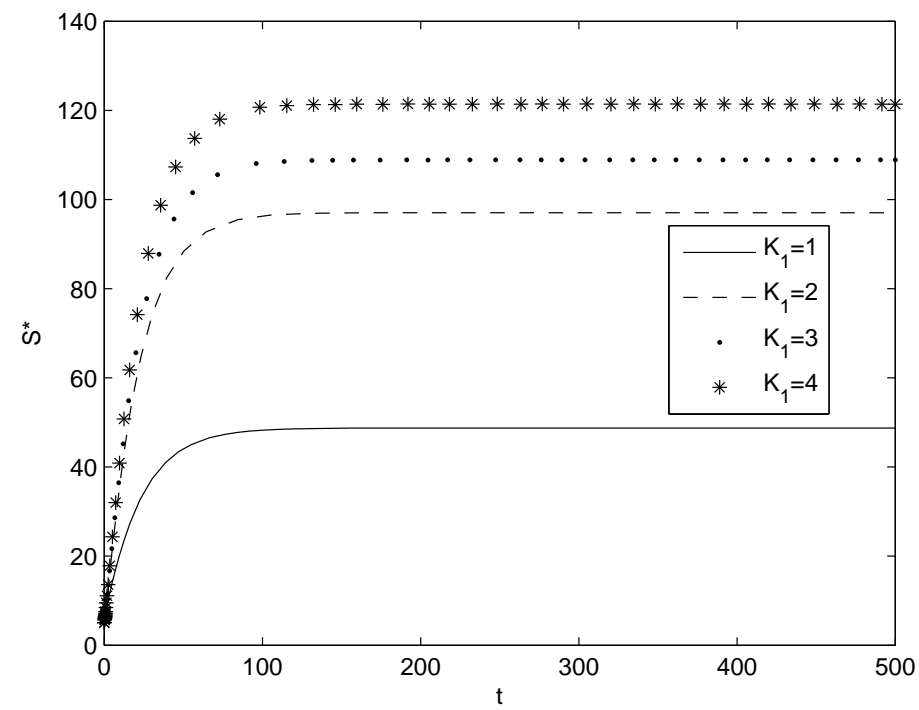

Figure 15. This figure shows the dependence of $R^{*}$ on the parameter $K_{1}$.

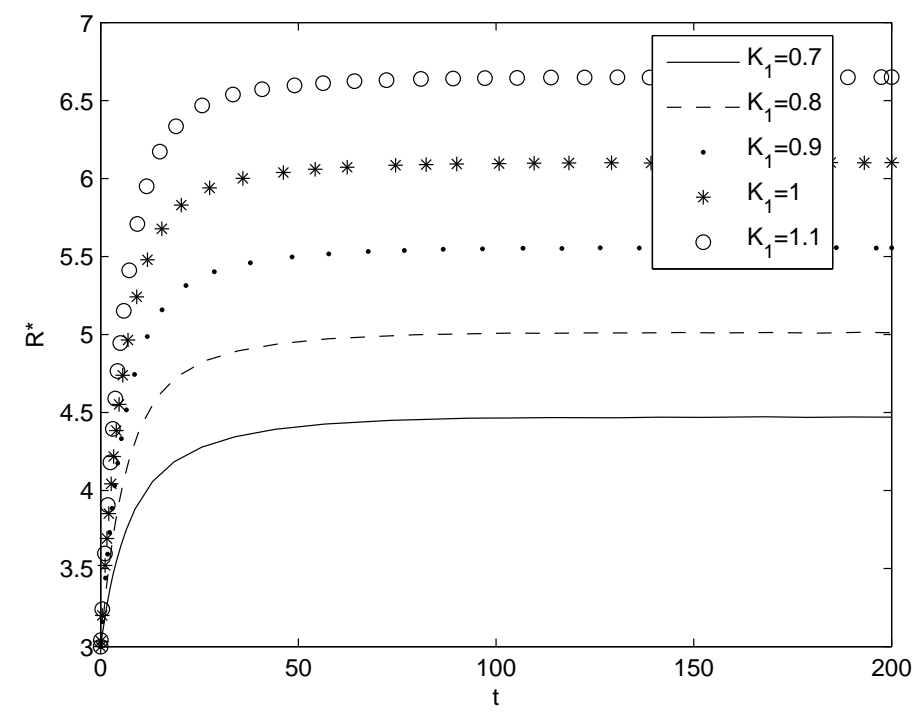

Figure 16. This figure shows the dependence of $S^{*}$ on the parameter $K_{1}$. 